\title{
Fission Product Yield Data Adjustment in a Prototype Version of TSURFER
}

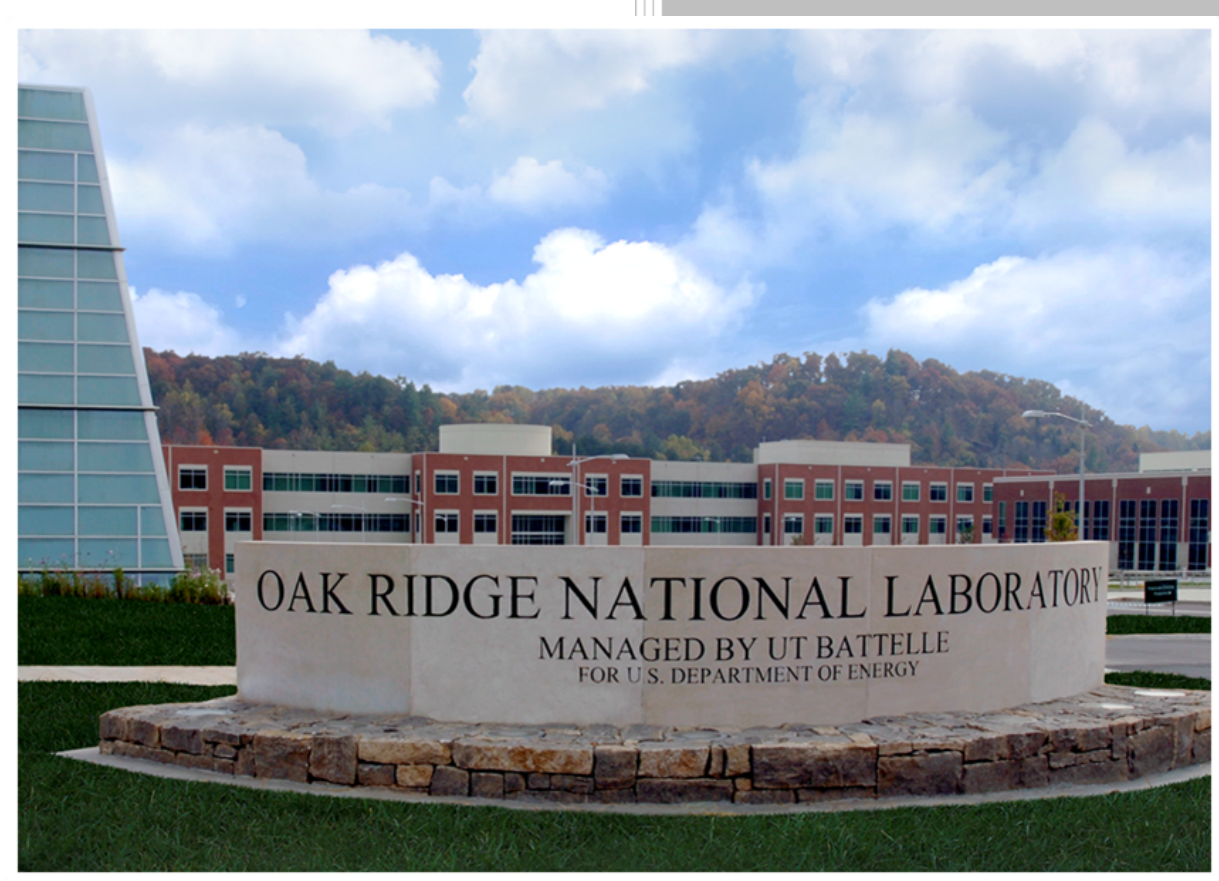

Keith C. Bledsoe

Marco T. Pigni

March 2021 


\section{DOCUMENT AVAILABILITY}

Reports produced after January 1, 1996, are generally available free via US Department of Energy (DOE) SciTech Connect.

Website www.osti.gov

Reports produced before January 1, 1996, may be purchased by members of the public from the following source:

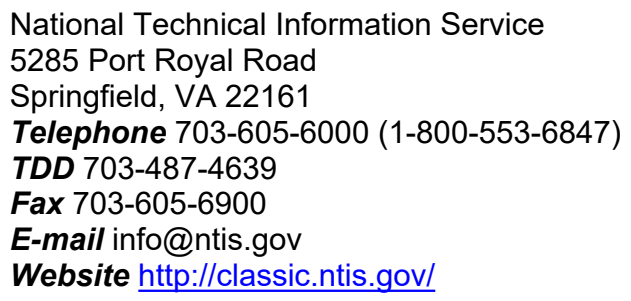

Reports are available to DOE employees, DOE contractors, Energy Technology Data Exchange representatives, and International Nuclear Information System representatives from the following source:

Office of Scientific and Technical Information

PO Box 62

Oak Ridge, TN 37831

Telephone 865-576-8401

Fax 865-576-5728

E-mail reports@osti.gov

Website http://www.osti.gov/contact.html

This report was prepared as an account of work sponsored by an agency of the United States Government. Neither the United States Government nor any agency thereof, nor any of their employees, makes any warranty, express or implied, or assumes any legal liability or responsibility for the accuracy, completeness, or usefulness of any information, apparatus, product, or process disclosed, or represents that its use would not infringe privately owned rights. Reference herein to any specific commercial product, process, or service by trade name, trademark, manufacturer, or otherwise, does not necessarily constitute or imply its endorsement, recommendation, or favoring by the United States Government or any agency thereof. The views and opinions of authors expressed herein do not necessarily state or reflect those of the United States Government or any agency thereof. 


\title{
Nuclear Nonproliferation Division
}

FISSION PRODUCT YIELD DATA ADJUSTMENT IN A PROTOTYPE VERSION OF TSURFER

\author{
Keith C. Bledsoe
}

Marco T. Pigni

March 2021

Prepared by

OAK RIDGE NATIONAL LABORATORY

Oak Ridge, TN 37831-6283

managed by

UT-BATTELLE, LLC

for the

US DEPARTMENT OF ENERGY

under contract DE-AC05-00OR22725 



\section{CONTENTS}

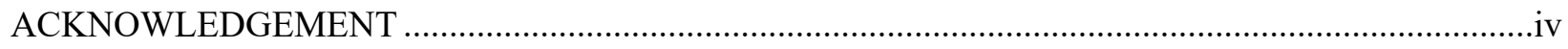

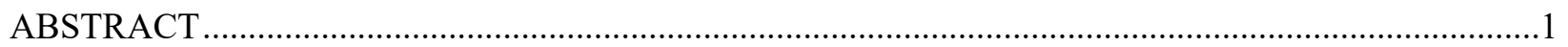

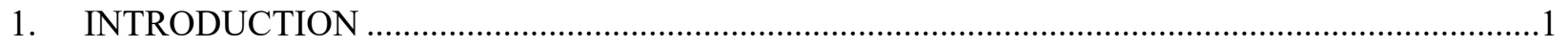

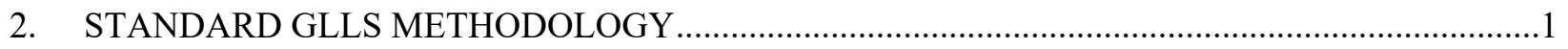

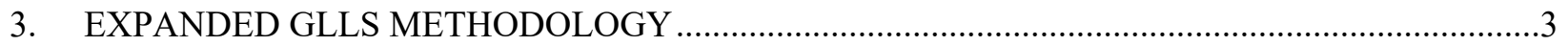

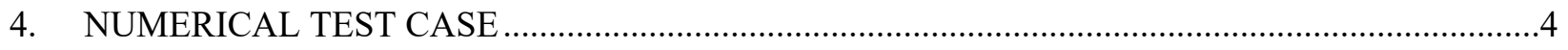

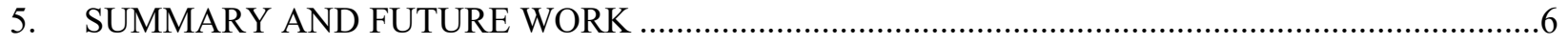

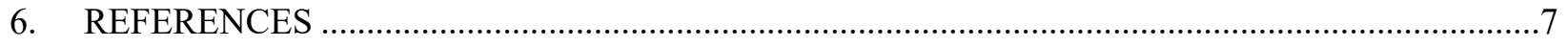




\section{ACKNOWLEDGMENT}

This research was sponsored by the Laboratory Directed Research and Development Program of Oak Ridge National Laboratory, managed by UT-Battelle LLC, for the US Department of Energy (LOIS ID 9436). 


\begin{abstract}
The TSURFER (Tool for Sensitivity/Uncertainty analysis of Response Functionals using Experimental Results) module of Oak Ridge National Laboratory's (ORNL's) SCALE code system has been updated to perform nuclear data adjustments for fixed-source irradiation/depletion problems. TSURFER uses a generalized linear least squares (GLLS) approach to consolidate a prior set of measured responses and corresponding calculated values to create the most self-consistent set of nuclear data. Traditionally, TSURFER adjustments have been performed for multigroup nuclear data such as reaction cross sections. In this work, TSURFER is expanded to perform adjustments to independent fission product yields and branching ratios that need equality constraints. To preserve equality constraints after the data adjustment procedure, an updated GLLS formulation includes a new Lagrange multiplier that forces data adjustment to sum to 0 for a given fission yield/branching ratio parent. A test problem illustrates that the newly updated TSURFER module satisfies the required constraint that adjustments for fission yield data sum to 0 .
\end{abstract}

\title{
1. INTRODUCTION
}

The TSURFER) module of the ORNL's SCALE code system [1] uses the generalized linear least squares (GLLS) approach to reduce discrepancies between the measured and calculated responses by adjusting nuclear data and experimental values [2]. Traditionally, TSURFER has only been applied to criticality experiments. As a part of the ORNL Laboratory Directed Research and Development project "Establishing the High Flux Isotope Reactor as a Unique Nuclear Data Measurement Facility," TSURFER was recently extended to perform data adjustment for fixed-source irradiation/depletion experiments. For these problems, maximum consistency between simulation and experiment requires the adjustment of fission product yield and branching ratio data. However, yields and branching ratios cannot be adjusted independently because they must satisfy equality constraints - the branching ratios for a given parent isotope must sum to 1.0, and the fission yields for a given parent isotope must sum to 2.0 (for a description of the fission yield sum see Ref. [3]). In this work we derive a new expression for the GLLS adjustment in which new Lagrange multiplier terms are introduced into the GLLS formulation to ensure that for a given parent, the sum of the adjustments is 0 . By ensuring the adjustment sum to 0 , we ensure that the equality constraints remain satisfied.

Section 2 reviews the standard TSURFER GLLS methodology, and Section 3 presents the new methodology that ensures that equality constraints are satisfied after the data adjustment. A numerical case to test the new GLLS methodology is reported in Section 4.

\section{STANDARD GLLS METHODOLOGY}

In this section we present a brief review of standard TSURFER GLLS methodology. This follow the derivation given in Ref. [2]. A more thorough review is given in Ref. [3 ]. The goal of the GLLS method in TSURFER is to adjust both the nuclear data from $\alpha$ to $\alpha^{\prime}$ and the measured integral responses from $m$ to $m^{\prime}$ such that they are most consistent with their respective uncertainty matrices, $C_{\alpha \alpha}$ and $C_{m m}$. According to the chi-squared metric $\left(\chi^{2}\right)$, this is done by minimizing the relation

$$
\chi^{2}=\Delta \alpha^{T} C_{\alpha \alpha}^{-1} \Delta \alpha+\Delta m^{T} C_{m m}^{-1} \Delta m,
$$

where $\Delta \alpha$ is an $n \times 1$ vector containing the adjustments to the $n$ nuclear data, $C_{\alpha \alpha}$ is an $n \times n$ covariance matrix of nuclear data, $\Delta m$ is an $I \times 1$ vector of adjustments to the $I$ measured responses, and $C_{m m}$ is an $I \times I$ covariance matrix. 
Let $k$ be an $I \times 1$ vector of calculated responses (in the GLLS process these will also be adjusted from $k$ to $\left.k^{\prime}\right)$. A unique solution to the minimization of $\chi^{2}$ can be obtained if we enforce the constraint that the adjusted calculated and measured responses agree $\left(k^{\prime}=m^{\prime}\right)$ and we satisfy the linearity condition $\Delta k=$ $S_{k \alpha} \Delta \alpha$, where $\Delta k$ is an $I \times 1$ vector of calculated response adjustments. The linearity condition is enforced using a Lagrange multiplier:

$$
\chi^{2}=\Delta \alpha^{T} C_{\alpha \alpha}^{-1} \Delta \alpha+\Delta m^{T} C_{m m}^{-1} \Delta m+2 \lambda_{1}\left(S_{k \alpha} \Delta \alpha-\Delta k\right) .
$$

The constraint that adjusted responses agree $\left(k^{\prime}=m^{\prime}\right)$ means that

$$
\Delta k=F_{m / k} \Delta m-d,
$$

where $F_{m / k}$ is a diagonal $I \times I$ matrix with ratios of $m_{i} / k_{i} ; i=1, \ldots, I$; and $d$ is the discrepancy vector

$$
d=\left\{\frac{k_{i}-m_{i}}{k_{i}}, i=1, \ldots, I\right.
$$

The linearity condition can therefore be expressed as $d=F_{m / k} \Delta m-S_{k \alpha} \Delta \alpha$, and $\chi^{2}$ can be written as

$$
\chi^{2}=\Delta \alpha^{T} C_{\alpha \alpha}^{-1} \Delta \alpha+\Delta m^{T} C_{m m}^{-1} \Delta m+2 \lambda_{1}\left(S_{k \alpha} \Delta \alpha-F_{m \backslash k} \Delta m+d\right) .
$$

To minimize $\chi^{2}$, we take the derivative of $\chi^{2}$ with respect to $\Delta \alpha$ and $\Delta m$ and set them to 0 :

$$
\begin{aligned}
& \frac{\partial \chi^{2}}{\partial \Delta \alpha}=0=2 C_{\alpha \alpha}^{-1} \Delta \alpha+2 S_{k \alpha}^{T} \lambda_{1}^{T}, \\
& \frac{\partial \chi^{2}}{\partial \Delta m}=0=2 C_{m m}^{-1} \Delta m-2 F_{\frac{m}{k}} \lambda_{1}^{T} .
\end{aligned}
$$

We will also use the relative uncertainty matrix of the discrepancy vector $d$, which can be obtained by standard propagation of error assuming no correlation between $k$ and $m$ :

$$
C_{d d}=S_{k \alpha} C_{\alpha \alpha} S_{k \alpha}^{T}+F_{m / k} C_{m m} F_{m / k}
$$

Here $S_{k \alpha}$ is a vector of sensitivities of response $k$ to nuclear data $\alpha$. This comes from a sensitivity analysis tool such as ORSEN, meaning all terms on the right side of Eq. (8) are known.

From Eq. (6) we have

$$
\Delta \alpha=-C_{\alpha \alpha} S_{k \alpha}^{T} \lambda_{1}^{T}
$$

and from Eq. (7) we have

$$
\Delta m=C_{m m} F_{m / k} \lambda_{1}^{T}
$$

Inserting Eqs. (9) and (10) into Eq. (8) yields

$$
C_{d d}=-S_{k \alpha} \Delta \alpha\left(\lambda_{1}^{T}\right)^{-1}+F_{m / k} \Delta m\left(\lambda_{1}^{T}\right)^{-1}, \quad \Rightarrow \lambda_{1}^{T}=C_{d d}^{-1}\left(F_{\frac{m}{k}} \Delta m-S_{k \alpha} \Delta \alpha\right) .
$$


Using the linearity condition, this becomes

$$
\lambda_{1}^{T}=C_{d d}^{-1} d
$$

We can now express $\Delta \alpha$ and $\Delta m$ in terms of known quantities:

$$
\begin{aligned}
& \Delta \alpha=-C_{\alpha \alpha} S_{k \alpha}^{T} C_{d d}^{-1} d \\
& \Delta m=C_{m m} F_{m / k} C_{d d}^{-1} d
\end{aligned}
$$

\section{EXPANDED GLLS METHODOLOGY}

Before GLLS adjustment, fission product yields for a given parent isotope sum to 2.0:

$$
\sum_{i} Y_{i}=2.0
$$

After GLLS adjustment, the fission product yields become $Y_{i}^{\prime}=Y_{i}+\Delta Y_{i}$. The sum of the updated fission product yields is

$$
\sum_{i} Y_{i}^{\prime}=\sum_{i} Y_{i}+\Delta Y_{i}=\sum_{i} Y_{i}+\sum_{i} \Delta Y_{i}=2.0+\sum_{i} \Delta Y_{i}
$$

Equation (16) shows that if the sum of adjustments equals 0 (i.e., $\sum_{i} \Delta Y_{i}=0$ ), then the adjusted fission product yields still sum to 2.0. Similarly, post-adjustment branching ratios will sum to 1.0 if the individual adjustments sum to 0 .

When we add a constraint that some of the nuclear data must sum to a certain constant (1.0 for branching ratio, 2.0 for fission yields), we add a second Lagrange multiplier to $\chi^{2}$ :

$$
\chi^{2}=\Delta \alpha^{T} C_{\alpha \alpha}^{-1} \Delta \alpha+\Delta m^{T} C_{m m}^{-1} \Delta m+2 \lambda_{1}\left(S_{k \alpha} \Delta \alpha-F_{m \backslash k} \Delta m+d\right)+2 \lambda_{2}(h \Delta \alpha)
$$

where $h$ is an $M \times n$ matrix and $M$ is the number of fission yield plus branching ratio parents. Each row of $h$ contains either $1 \mathrm{~s}$ or $0 \mathrm{~s}$. The constraint that $h \Delta \alpha=0$ forces the adjustments for fission yields and branching ratios to sum to 0 , thus maintaining their initial constraints (i.e., if the prior values summed to 2.0, the adjusted values will still sum to 2.0).

The derivatives of $\chi^{2}$ become

$$
\frac{\partial \chi^{2}}{\partial \Delta \alpha}=0=2 C_{\alpha \alpha}^{-1} \Delta \alpha+2 S_{k \alpha}^{T} \lambda_{1}^{T}+2 h^{T} \lambda_{2}^{T}
$$

and

$$
\frac{\partial \chi^{2}}{\partial \Delta m}=0=2 C_{m m}^{-1} \Delta m+2 F_{m / k} \lambda_{1}^{T} .
$$

Solving Eq. (18) for $\Delta \alpha$ yields

$$
\Delta \alpha=-C_{\alpha \alpha}\left(S_{k \alpha}^{T} \lambda_{1}^{T}+h^{T} \lambda_{2}^{T}\right),
$$

and solving Eq. (19) for $\Delta m$ yields 


$$
\Delta m=C_{m m} F_{m / k} \lambda_{1}^{T}
$$

From Eq. (20), we get

$$
C_{\alpha \alpha} S_{k \alpha}^{T}=\left(\Delta \alpha+h^{T} \lambda_{2}^{T}\right)\left(\lambda_{1}^{T}\right)^{-1}
$$

and from Eq. (21) we get

$$
C_{m m} F_{m / k}=\Delta m\left(\lambda_{1}^{T}\right)^{-1}
$$

Inserting these results into Eq. (8) yields

$$
C_{d d}=-S_{k \alpha}\left(\Delta \alpha+h^{T} \lambda_{2}^{T}\right)\left(\lambda_{1}^{T}\right)^{-1}+F_{m / k} \Delta m\left(\lambda_{1}^{T}\right)^{-1}
$$

From the linearity condition $F_{m / k} \Delta m-\Delta k=F_{m / k} \Delta m-S_{k \alpha}^{T} \Delta \alpha=d$, we get

$$
\begin{gathered}
C_{d d}=\left(d-S_{k \alpha} h^{T} \lambda_{2}^{T}\right)\left(\lambda_{1}^{T}\right)^{-1} \\
\Rightarrow \lambda_{1}^{T}=C_{d d}^{-1}\left(d-S_{k \alpha} h^{T} \lambda_{2}^{T}\right) .
\end{gathered}
$$

Now using the condition that $h \Delta \alpha=0$,

$$
\begin{gathered}
h \Delta \alpha=0=-h C_{a a}\left(S_{k \alpha}^{T} \lambda_{1}^{T}+h^{T} \lambda_{2}^{T}\right)=-h C_{a a}\left(S_{k \alpha}^{T} C_{d d}^{-1}\left(d-S_{k \alpha} h^{T} \lambda_{2}^{T}\right)+h^{T} \lambda_{2}^{T}\right) \\
=-h C_{a a} S_{k \alpha}^{T} C_{d d}^{-1} d+h C_{a a} S_{k a}^{T} C_{d d}^{-1} S_{k \alpha} h^{T} \lambda_{2}^{T}-h C_{\alpha \alpha} h^{T} \lambda_{2}^{T} .
\end{gathered}
$$

(Note, $C_{a a} S_{k \alpha}^{T} C_{d d}^{-1} d$ is the adjustment $\Delta \alpha$ for the unconstrained problem.) Solving for $\lambda_{2}^{T}$ yields

$$
\lambda_{2}^{T}=\left(h C_{a a} S_{k \alpha}^{T} C_{d d}^{-1} S_{k \alpha} h^{T}-h C_{\alpha \alpha} h^{T}\right)^{-1} h C_{\alpha \alpha} S_{k \alpha}^{T} C_{d d}^{-1} d
$$

$\lambda_{2}^{T}$ is now expressed in terms of known quantities. With this, we can determine $\lambda_{1}, \Delta \alpha$, and $\Delta m$.

Note, the possibility for simplification is based on the form of the covariance matrix of the data. If $C_{\alpha \alpha}$ has rows and columns that sum to 0 (in the locations where $h$ contains $1 \mathrm{~s}$ ) then $h^{T} C_{\alpha \alpha}=0$ and thus $\lambda_{2}$ $=0, \lambda_{1}=C_{d d}^{-1} d$. The adjustments for $\Delta \alpha$ and $\Delta m$ are the same as in standard TSURFER. This row/column sum zero approach has been used for equality-constrained GLLS in the past [5], but as noted in Ref. [4], obtaining a covariance matrix of this form for fission product yields is generally not possible.

\section{NUMERICAL TEST CASE}

The procedure for obtaining covariance matrices for independent fission product yields and related constraints is detailed in Refs. [4,6]. Here those covariance matrices are used with the updated GLLS approach to perform fission product yield data adjustment using "measured" data from two simulated irradiation experiments. Fission yield covariance data are currently available at three incident neutron energies, $0.0253,500$, and $1.4 \times 10^{7} \mathrm{eV}$; however, in these calculations we used only the $500 \mathrm{eV}$ covariance data. In future work we will include extrapolation between files to generate covariance data to match the average fission energy of the simulation. 
For this test case, two experiments were simulated using the ORIGEN module of SCALE. In the first simulation (Experiment 1) a mixture of $1,000 \mathrm{~g}$ of ${ }^{235} \mathrm{U}$ and $500 \mathrm{~g}$ of ${ }^{238} \mathrm{~Np}$ was irradiated for 12 hours with a flux of $3.77 \times 10^{12} \mathrm{n} / \mathrm{cm}^{2} \mathrm{~s}$ and allowed to decay for 12 hours. In the second simulation (Experiment 2), a 1,000 g sample of ${ }^{235} \mathrm{U}$ was irradiated for 12 hours with the same flux and allowed to decay for 12 hours. The amount of ${ }^{135} \mathrm{Xe}$ was the quantity measured at the end of the decay time. At the end of the Experiment 1, $0.00175 \mathrm{~g}$ of ${ }^{135} \mathrm{Xe}$ was present, and at the end the Experiment 2, $0.00068 \mathrm{~g}$ of ${ }^{135} \mathrm{Xe}$ was present. Measured values were then created by adding a random noise term to these simulated values. This resulted in "measured" values of $0.00161 \mathrm{~g}$ of ${ }^{135} \mathrm{Xe}$ for Experiment 1 and $0.00071 \mathrm{~g}$ of ${ }^{135} \mathrm{Xe}$ for the Experiment 2.

The sensitivities of the final ${ }^{135} \mathrm{Xe}$ masses with respect to fission yield data were calculated using the depletion perturbation theory method in a prototype ORIGEN sensitivity module [7]. The largest sensitivities are given in Table 1 .

Table 1. Sensitivities of final mass ${ }^{135} \mathrm{Xe}$ to fission product yields.

\begin{tabular}{|c|c|c|}
\hline Reaction & $\begin{array}{c}\text { Experiment 1 } \\
\text { Sensitivities }\end{array}$ & $\begin{array}{c}\text { Experiment 2 } \\
\text { Sensitivities }\end{array}$ \\
\hline${ }^{235} \mathrm{U} \rightarrow{ }^{135} \mathrm{Sb}$ & $7.04 \times 10^{-3}$ & $1.81 \times 10^{-2}$ \\
${ }^{235} \mathrm{U} \rightarrow{ }^{135} \mathrm{Te}$ & $1.87 \times 10^{-1}$ & $4.81 \times 10^{-1}$ \\
${ }^{235} \mathrm{U} \rightarrow{ }^{135} \mathrm{I}$ & $1.74 \times 10^{-1}$ & $4.48 \times 10^{-1}$ \\
${ }^{235} \mathrm{U} \rightarrow{ }^{135} \mathrm{Xe}$ & $1.26 \times 10^{-2}$ & $3.23 \times 10^{-2}$ \\
${ }^{235} \mathrm{U} \rightarrow{ }^{136} \mathrm{Sn}$ & $7.64 \times 10^{-7}$ & $1.96 \times 10^{-6}$ \\
${ }^{235} \mathrm{U} \rightarrow{ }^{136} \mathrm{Sb}$ & $3.41 \times 10^{-4}$ & $8.76 \times 10^{-4}$ \\
${ }^{235} \mathrm{U} \rightarrow{ }^{136} \mathrm{Te}$ & $1.04 \times 10^{-4}$ & $2.66 \times 10^{-3}$ \\
${ }^{235} \mathrm{U} \rightarrow{ }^{137} \mathrm{Sb}$ & $5.45 \times 10^{-7}$ & $1.40 \times 10^{-6}$ \\
${ }^{235} \mathrm{U} \rightarrow{ }^{137} \mathrm{Sn}$ & $5.85 \times 10^{-5}$ & $1.50 \times 10^{-4}$ \\
${ }^{238} \mathrm{~Np} \rightarrow{ }^{135} \mathrm{Sb}$ & $2.27 \times 10^{-2}$ & $<10^{-10}$ \\
${ }^{238} \mathrm{~Np} \rightarrow{ }^{135} \mathrm{Te}$ & $3.36 \times 10^{-1}$ & $<10^{-10}$ \\
${ }^{238} \mathrm{~Np} \rightarrow{ }^{135} \mathrm{I}$ & $2.40 \times 10^{-1}$ & $<10^{-10}$ \\
${ }^{238} \mathrm{~Np} \rightarrow{ }^{135} \mathrm{Xe}$ & $1.68 \times 10^{-3}$ & $<10^{-10}$ \\
${ }^{238} \mathrm{~Np} \rightarrow{ }^{136} \mathrm{Sn}$ & $3.33 \times 10^{-6}$ & $<10^{-10}$ \\
${ }^{238} \mathrm{~Np} \rightarrow{ }^{136} \mathrm{Sb}$ & $2.27 \times 10^{-3}$ & $<10^{-10}$ \\
${ }^{238} \mathrm{~Np} \rightarrow{ }^{136} \mathrm{Te}$ & $2.49 \times 10^{-3}$ & $<10^{-10}$ \\
${ }^{238} \mathrm{~Np} \rightarrow{ }^{137} \mathrm{Sb}$ & $4.17 \times 10^{-8}$ & $<10^{-10}$ \\
${ }^{238} \mathrm{~Np} \rightarrow{ }^{137} \mathrm{Sn}$ & $5.12 \times 10^{-6}$ & $<10^{-10}$ \\
${ }^{238} \mathrm{Pu} \rightarrow{ }^{135} \mathrm{Sb}$ & $7.27 \times 10^{-6}$ & $<10^{-10}$ \\
${ }^{238} \mathrm{Pu} \rightarrow{ }^{135} \mathrm{Te}$ & $3.63 \times 10^{-4}$ & $<10^{-10}$ \\
${ }^{238} \mathrm{Pu} \rightarrow{ }^{135} \mathrm{I}$ & $6.55 \times 10^{-4}$ & $<10^{-10}$ \\
${ }^{238} \mathrm{Pu} \rightarrow{ }^{135} \mathrm{Xe}$ & $3.39 \times 10^{-5}$ & $<10^{-10}$ \\
${ }^{238} \mathrm{Pu} \rightarrow{ }^{136} \mathrm{Sb}$ & $3.90 \times 10^{-7}$ & \\
${ }^{238} \mathrm{Pu} \rightarrow{ }^{136} \mathrm{Te}$ & $1.67 \times 10^{-6}$ & \\
\hline
\end{tabular}

Table 2 shows the absolute adjusted values for fission product yields. Note, for the individual parent isotopes, the adjustments sum to 0 as required. 
Table 2. Absolute adjusted value of fission product yields.

\begin{tabular}{|c|c|}
\hline Fission Yield & Adjustment \\
\hline \multicolumn{2}{|c|}{${ }^{235} \mathrm{U}$ Fission Yields } \\
\hline${ }^{235} \mathrm{U} \rightarrow{ }^{135} \mathrm{Sb}$ & 0.2414044 \\
\hline${ }^{235} \mathrm{U} \rightarrow{ }^{135} \mathrm{Te}$ & -0.1862993 \\
\hline${ }^{235} \mathrm{U} \rightarrow{ }^{135} \mathrm{I}$ & -0.0889851 \\
\hline${ }^{235} \mathrm{U} \rightarrow{ }^{135} \mathrm{Xe}$ & 0.0175893 \\
\hline${ }^{235} \mathrm{U} \rightarrow{ }^{136} \mathrm{Sn}$ & 0.0025497 \\
\hline${ }^{235} \mathrm{U} \rightarrow{ }^{136} \mathrm{Sb}$ & 0.0103145 \\
\hline${ }^{235} \mathrm{U} \rightarrow{ }^{136} \mathrm{Te}$ & -0.0013855 \\
\hline${ }^{235} \mathrm{U} \rightarrow{ }^{137} \mathrm{Sb}$ & 0.0025287 \\
\hline${ }^{235} \mathrm{U} \rightarrow{ }^{137} \mathrm{Sn}$ & 0.0022834 \\
\hline Sum of Adjustments & 0.0000000 \\
\hline \multicolumn{2}{|c|}{${ }^{238} \mathrm{~Np}$ Fission Yields } \\
\hline${ }^{238} \mathrm{~Np} \rightarrow{ }^{135} \mathrm{Sb}$ & 0.0502742 \\
\hline${ }^{238} \mathrm{~Np} \rightarrow{ }^{135} \mathrm{Te}$ & -0.1670862 \\
\hline${ }^{238} \mathrm{~Np} \rightarrow{ }^{135} \mathrm{I}$ & -0.0139868 \\
\hline${ }^{238} \mathrm{~Np} \rightarrow{ }^{135} \mathrm{Xe}$ & 0.0221285 \\
\hline${ }^{238} \mathrm{~Np} \rightarrow{ }^{136} \mathrm{Sn}$ & 0.0206014 \\
\hline${ }^{238} \mathrm{~Np} \rightarrow{ }^{136} \mathrm{Sb}$ & 0.0230141 \\
\hline${ }^{238} \mathrm{~Np} \rightarrow{ }^{136} \mathrm{Te}$ & 0.0239119 \\
\hline${ }^{238} \mathrm{~Np} \rightarrow{ }^{137} \mathrm{Sb}$ & 0.0206002 \\
\hline${ }^{238} \mathrm{~Np} \rightarrow{ }^{137} \mathrm{Sn}$ & 0.0205426 \\
\hline Sum of Adjustments & 0.0000000 \\
\hline \multicolumn{2}{|c|}{${ }^{238}$ Pu Fission Yields } \\
\hline${ }^{238} \mathrm{Pu} \rightarrow{ }^{135} \mathrm{Sb}$ & 0.0001254 \\
\hline${ }^{238} \mathrm{Pu} \rightarrow{ }^{135} \mathrm{Te}$ & -0.0000690 \\
\hline${ }^{238} \mathrm{Pu} \rightarrow{ }^{135} \mathrm{I}$ & -0.0004430 \\
\hline${ }^{238} \mathrm{Pu} \rightarrow{ }^{135} \mathrm{Xe}$ & 0.0001337 \\
\hline${ }^{238} \mathrm{Pu} \rightarrow{ }^{136} \mathrm{Sb}$ & 0.0001215 \\
\hline${ }^{238} \mathrm{Pu} \rightarrow{ }^{136} \mathrm{Te}$ & 0.0001313 \\
\hline Sum of Adjustments & 0.0000000 \\
\hline
\end{tabular}

\section{SUMMARY AND FUTURE WORK}

A new prototype version of SCALE's TSURFER module has been created to perform nuclear data adjustments for fixed source irradiation/depletion problems. This update was needed because fission product yields and branching ratios must satisfy equality constraints after the adjustment. By introducing a new Lagrange multiplier term into the GLLS method used by TSURFER, we ensured that the equality constraints remain satisfied. This was verified using a numerical example.

Branching ratio covariance data are not currently available and thus could not be used in the numerical example. Generating this data will be the subject of future work. 


\section{REFERENCES}

1. W. A. Wieselquist, R. A. Lefebvre, and M. A. Jessee, Eds., SCALE Code System, ORNL/TM2005/39, Version 6.2.4, Oak Ridge National Laboratory, Oak Ridge, TN (2020).

2. J. J. Wagschal and Y. Yeivin, "Significance of Lagrange Multipliers in Cross-Section Adjustment," No. CONF-800607-49, Oak Ridge National Laboratory (1980).

3. M. L. Williams, B. L. Broadhead, M. A. Jessee, J. J. Wagschal, and R. A. Lefebvre, “TSURFER: An Adjustment Code to Determine Biases and Uncertainties in Nuclear System Responses by Consolidating Differential Data and Benchmark Integral Experiments," SCALE 6.2 Manual, Sect. 6.6, ORNL/TM-2005/39, Oak Ridge National Laboratory (2016).

4. M. T. Pigni and K. C. Bledsoe, Standardizing a Renewed Fission Product Yield Library and Related Covariances (Part I), ORNL/TM-2020/1660, Oak Ridge National Laboratory (2020).

5. K. C. Bledsoe, M. A. Jessee, and J. A. Favorite, "Application of Generalized Linear Least-Squares for Uncertainty Analysis in Inverse Transport Problems," Trans. Am. Nucl. Soc., 108, 445-448 (2013).

6. M. T. Pigni, M. W. Francis, I. C. Gauld, "Investigation of Inconsistent ENDF/B-BII.1 Independent and Cumulative Fission Product Yields with Proposed Revision," Nuclear Data Sheets, 123, 231 (2015).

7. K. C. Bledsoe, G. Ilas, and S. L. Hogle, "Application of Depletion Perturbation Theory for Sensitivity Analysis in the High Flux Isotope Reactor," Trans. Am. Nucl. Soc., 121, 1453-1456 (2019). 